\title{
REAL HYPERSURFACES OF TYPE $B$ IN COMPLEX TWO-PLANE GRASSMANNIANS RELATED TO THE REEB VECTOR
}

\author{
Hyunjin Lee And Young Jin Suh
}

\begin{abstract}
In this paper we give a new characterization of real hypersurfaces of type $B$, that is, a tube over a totally geodesic $\mathbb{Q} P^{n}$ in complex two-plane Grassmannians $G_{2}\left(\mathbb{C}^{m+2}\right)$, where $m=2 n$, with the Reeb vector $\xi$ belonging to the distribution $\mathfrak{D}$, where $\mathfrak{D}$ denotes a subdistribution in the tangent space $T_{x} M$ such that $T_{x} M=\mathfrak{D} \oplus \mathfrak{D}^{\perp}$ for any point $x \in M$ and $\mathfrak{D}^{\perp}=\operatorname{Span}\left\{\xi_{1}, \xi_{2}, \xi_{3}\right\}$.
\end{abstract}

\section{Introduction}

The study of real hypersurfaces in non-flat complex space forms or quaternionic space forms is a classical topic in differential geometry. For instance, there have been many investigations for homogeneous hypersurfaces of type $A_{1}, A_{2}, B, C, D$ and $E$ in complex projective space $\mathbb{C} P^{m}$. They are completely classified by Berndt [2], Cecil and Ryan [5], Kimura [7] and Takagi [10]. Here, explicitly, we mention that $A_{1}$ : geodesic hyperspheres, $A_{2}$ : a tube around a totally geodesic complex projective spaces $\mathbb{C} P^{k}, B$ : a tube around a complex quadric $Q^{m-1}$ and can be viewed as a tube around a real projective space $\mathbb{R} P^{m}, C$ : a tube around the Segre embedding of $\mathbb{C} P^{1} \times \mathbb{C} P^{k}$ into $\mathbb{C} P^{2 k+1}$ for some $k \geq 2, D$ : a tube around the Plücker embedding into $\mathbb{C} P^{9}$ of the complex Grassmannian manifold $G_{2}\left(\mathbb{C}^{5}\right)$ of complex 2-planes in $\mathbb{C}^{5}$ and $E$ : a tube around the half spin embedding into $\mathbb{C} P^{15}$ of the Hermitian symmetric space $S O(10) / U(5)$.

But until now there were only a few characterizations of homogeneous real hypersurfaces of type $B$, that is, a tube over a real projective space $\mathbb{R} P^{m}$ in complex projective space $\mathbb{C} P^{m}$. Among them, Yano and Kon [11] gave a

Received December 11, 2008; Revised August 11, 2009.

2000 Mathematics Subject Classification. Primary 53C40; Secondary 53C15.

Key words and phrases. complex two-plane Grassmannians, real hypersurfaces of type $B$, Hopf hypersurface, Reeb vector field, $\mathfrak{D}$-distribution.

This work was supported by grant Proj. No. R17-2008-001-01001-0 from National Research Foundation. 
characterization for real hypersurfaces of type $B$ in $\mathbb{C} P^{m}$ in such a way that $A \phi+\phi A=k \phi$, where $k$ is non-zero constant.

Now let us consider a complex two-plane Grassmannian $G_{2}\left(\mathbb{C}^{m+2}\right)$ which consists of all complex 2-dimensional linear subspaces in $\mathbb{C}^{m+2}$. This Riemannian symmetric space $G_{2}\left(\mathbb{C}^{m+2}\right)$ has a remarkable geometrical structure. It is the unique compact irreducible Riemannian manifold being equipped with both a Kähler structure $J$ and a quaternionic Kähler structure $\mathfrak{J}$ not containing $J$. In other words, $G_{2}\left(\mathbb{C}^{m+2}\right)$ is the unique compact, irreducible, Kähler, quaternionic Kähler manifold which is not a hyper Kähler manifold (See Berndt and Suh [3], [4]). So, in $G_{2}\left(\mathbb{C}^{m+2}\right)$ we have the two natural geometrical conditions for real hypersurfaces $M$ that $[\xi]=\operatorname{Span}\{\xi\}$ or $\mathfrak{D}^{\perp}=\operatorname{Span}\left\{\xi_{1}, \xi_{2}, \xi_{3}\right\}$ are invariant under the shape operator $A$ of $M$.

The almost contact structure vector field $\xi$ mentioned above is defined by $\xi=-J N$, where $N$ denotes a local unit normal vector field of $M$ in $G_{2}\left(\mathbb{C}^{m+2}\right)$ and it is said to be a Reeb vector field. The almost contact three structure vector fields $\left\{\xi_{1}, \xi_{2}, \xi_{3}\right\}$ are defined by $\xi_{\nu}=-J_{\nu} N, \nu=1,2,3$, where $J_{\nu}$ denotes a canonical local basis of a quaternionic Kähler structure $\mathfrak{J}$.

By using two invariant structures for the Reeb vector field $\xi$ and the distribution $\mathfrak{D}^{\perp}=\operatorname{Span}\left\{\xi_{1}, \xi_{2}, \xi_{3}\right\}$, Berndt and the second author [3] have proved the following:

Theorem A. Let $M$ be a connected orientable real hypersurface in $G_{2}\left(\mathbb{C}^{m+2}\right)$, $m \geq 3$. Then both $[\xi]$ and $\mathfrak{D}^{\perp}$ are invariant under the shape operator $A$ of $M$ if and only if

(A) $M$ is an open part of a tube around a totally geodesic $G_{2}\left(\mathbb{C}^{m+1}\right)$ in $G_{2}\left(\mathbb{C}^{m+2}\right)$, or

(B) $m$ is even, say $m=2 n$, and $M$ is an open part of a tube around a totally geodesic $\mathbb{Q} P^{n}$ in $G_{2}\left(\mathbb{C}^{m+2}\right)$.

When the Reeb flow on $M$ in $G_{2}\left(\mathbb{C}^{m+2}\right)$ is isometric, we say that the Reeb vector field $\xi$ on $M$ is Killing. Moreover, the Reeb vector field $\xi$ is said to be Hopf if it is invariant by the shape operator $A$. The 1-dimensional foliation of $M$ by the integral manifolds of the Reeb vector field $\xi$ is said to be a Hopf foliation of $M$. We say that $M$ is a Hopf hypersurface in $G_{2}\left(\mathbb{C}^{m+2}\right)$ if and only if the Hopf foliation of $M$ is totally geodesic. By the formulas in Section 2 it can be easily checked that $M$ is Hopf if and only if the Reeb vector field $\xi$ is Hopf.

In particular, the second author [8] gave a characterization of type $B$ among Hopf hypersurfaces in $G_{2}\left(\mathbb{C}^{m+2}\right)$ when the almost contact 3 -structure tensors $\left\{\phi_{1}, \phi_{2}, \phi_{3}\right\}$ commute with the shape operator $A$ on the orthogonal complement of the one dimensional distribution $[\xi]$. Moreover, he also gave another characterization of real hypersurfaces of type $B$ in $G_{2}\left(\mathbb{C}^{m+2}\right)$ in terms of contact hypersurface, that is, $A \phi+\phi A=k \phi$, where $k$ is non-zero constant (See $[9])$. 
On the other hand, it can be easily seen that the Reeb vector $\xi$ for real hypersurfaces of type $B$ in Theorem A belongs to the distribution $\mathfrak{D}$ (See [2]). Then naturally we are able to consider a converse problem. It should be an interesting problem to check that whether a real hypersurface of type $B$, that is, a tube around a totally geodesic $\mathbb{Q} P^{n}, m=2 n$, in $G_{2}\left(\mathbb{C}^{m+2}\right)$, is only a hypersurface with its Reeb vector $\xi$ belonging to the distribution $\mathfrak{D}$.

From such a view point, we affirmatively answer for this problem. In this paper we give a new characterization of real hypersurfaces of type $B$ in $G_{2}\left(\mathbb{C}^{m+2}\right)$ as follows:

Main Theorem. Let $M$ be a connected orientable Hopf real hypersurface in $G_{2}\left(\mathbb{C}^{m+2}\right), m \geq 3$. Then the Reeb vector $\xi$ belongs to the distribution $\mathfrak{D}$ if and only if $M$ is locally congruent to an open part of a tube around a totally geodesic $\mathbb{Q} P^{n}$ in $G_{2}\left(\mathbb{C}^{m+2}\right)$, where $m=2 n$.

\section{Riemannian geometry of $G_{2}\left(\mathbb{C}^{m+2}\right)$}

In this section we summarize basic material about $G_{2}\left(\mathbb{C}^{m+2}\right)$, for details we refer to [1], [3] and [4]. By $G_{2}\left(\mathbb{C}^{m+2}\right)$ we denote the set of all complex twodimensional linear subspaces in $\mathbb{C}^{m+2}$. The special unitary group $G=S U(m+$ $2)$ acts transitively on $G_{2}\left(\mathbb{C}^{m+2}\right)$ with stabilizer isomorphic to $K=S(U(2) \times$ $U(m)) \subset G$. Then $G_{2}\left(\mathbb{C}^{m+2}\right)$ can be identified with the homogeneous space $G / K$, which we equip with the unique analytic structure for which the natural action of $G$ on $G_{2}\left(\mathbb{C}^{m+2}\right)$ becomes analytic. Denote by $\mathfrak{g}$ and $\mathfrak{k}$ the Lie algebra of $G$ and $K$, respectively, and by $\mathfrak{m}$ the orthogonal complement of $\mathfrak{k}$ in $\mathfrak{g}$ with respect to the Cartan-Killing form $B$ of $\mathfrak{g}$. Then $\mathfrak{g}=\mathfrak{k} \oplus \mathfrak{m}$ is an $A d(K)$-invariant reductive decomposition of $\mathfrak{g}$. We put $o=e K$ and identify $T_{o} G_{2}\left(\mathbb{C}^{m+2}\right)$ with $\mathfrak{m}$ in the usual manner. Since $B$ is negative definite on $\mathfrak{g}$, its negative restricted to $\mathfrak{m} \times \mathfrak{m}$ yields a positive definite inner product on $\mathfrak{m}$. By $A d(K)$-invariance of $B$ this inner product can be extended to a $G$-invariant Riemannian metric $g$ on $G_{2}\left(\mathbb{C}^{m+2}\right)$. In this way $G_{2}\left(\mathbb{C}^{m+2}\right)$ becomes a Riemannian homogeneous space, even a Riemannian symmetric space. For computational reasons we normalize $g$ such that the maximal sectional curvature of $\left(G_{2}\left(\mathbb{C}^{m+2}\right), g\right)$ is eight.

When $m=1, G_{2}\left(\mathbb{C}^{3}\right)$ is isometric to the two-dimensional complex projective space $\mathbb{C} P^{2}$ with constant holomorphic sectional curvature eight. When $m=2$, we note that the isomorphism $\operatorname{Spin}(6) \simeq S U(4)$ yields an isometry between $G_{2}\left(\mathbb{C}^{4}\right)$ and the real Grassmann manifold $G_{2}^{+}\left(\mathbb{R}^{6}\right)$ of oriented two-dimensional linear subspaces of $\mathbb{R}^{6}$. In this paper, we will assume $m \geq 3$.

The Lie algebra $\mathfrak{k}$ has the direct sum decomposition, that is, a Cartan decomposition

$$
\mathfrak{k}=\mathfrak{s} u(m) \oplus \mathfrak{s} u(2) \oplus \mathfrak{R},
$$

where $\mathfrak{R}$ denotes the center of $\mathfrak{k}$. Viewing $\mathfrak{k}$ as the holonomy algebra of $G_{2}\left(\mathbb{C}^{m+2}\right)$, the center $\mathfrak{R}$ induces a Kähler structure $J$ and the $\mathfrak{s} u(2)$-part a quaternionic Kähler structure $\mathfrak{J}$ on $G_{2}\left(\mathbb{C}^{m+2}\right)$. If $J_{\nu}$ is any almost Hermitian structure in $\mathfrak{J}$, then $J J_{\nu}=J_{\nu} J$, and $J J_{\nu}$ is a symmetric endomorphism with 
$\left(J J_{\nu}\right)^{2}=I$ and $\operatorname{tr}\left(J J_{\nu}\right)=0$. This fact will be used frequently throughout this paper.

A canonical local basis $J_{1}, J_{2}, J_{3}$ of $\mathfrak{J}$ consists of three local almost Hermitian structures $J_{\nu}$ in $\mathfrak{J}$ such that $J_{\nu} J_{\nu+1}=J_{\nu+2}=-J_{\nu+1} J_{\nu}$, where the index is taken modulo three. Since $\mathfrak{J}$ is parallel with respect to the Riemannian connection $\bar{\nabla}$ of $\left(G_{2}\left(\mathbb{C}^{m+2}\right), g\right)$, there exist for any canonical local basis $J_{1}, J_{2}, J_{3}$ of $\mathfrak{J}$ three local one-forms $q_{1}, q_{2}, q_{3}$ such that

$$
\bar{\nabla}_{X} J_{\nu}=q_{\nu+2}(X) J_{\nu+1}-q_{\nu+1}(X) J_{\nu+2}
$$

for all vector fields $X$ on $G_{2}\left(\mathbb{C}^{m+2}\right)$.

Let $p \in G_{2}\left(\mathbb{C}^{m+2}\right)$ and $W$ a subspace of $T_{p} G_{2}\left(\mathbb{C}^{m+2}\right)$. We say that $W$ is a quaternionic subspace of $T_{p} G_{2}\left(\mathbb{C}^{m+2}\right)$ if $J W \subset W$ for all $J \in \mathfrak{J}_{p}$. And we say that $W$ is a totally complex subspace of $T_{p} G_{2}\left(\mathbb{C}^{m+2}\right)$ if there exists a one-dimensional subspace $\mathfrak{V}$ of $\mathfrak{J}_{p}$ such that $J W \subset W$ for all $J \in \mathfrak{V}$ and $J W \perp W$ for all $J \in \mathfrak{V}^{\perp} \subset \mathfrak{J}_{p}$. Here, the orthogonal complement of $\mathfrak{V}$ in $\mathfrak{J}_{p}$ is taken with respect to the bundle metric and orientation on $\mathfrak{J}$ for which any local oriented orthonormal frame field of $\mathfrak{J}$ is a canonical local basis of $\mathfrak{J}$. A quaternionic (resp. totally complex) submanifold of $G_{2}\left(\mathbb{C}^{m+2}\right)$ is a submanifold all of whose tangent spaces are quaternionic (resp. totally complex) subspaces of the corresponding tangent spaces of $G_{2}\left(\mathbb{C}^{m+2}\right)$.

The Riemannian curvature tensor $\bar{R}$ of $G_{2}\left(\mathbb{C}^{m+2}\right)$ is locally given by

$$
\begin{aligned}
\bar{R}(X, Y) Z= & g(Y, Z) X-g(X, Z) Y+g(J Y, Z) J X \\
& -g(J X, Z) J Y-2 g(J X, Y) J Z \\
& +\sum_{\nu=1}^{3}\left\{g\left(J_{\nu} Y, Z\right) J_{\nu} X-g\left(J_{\nu} X, Z\right) J_{\nu} Y-2 g\left(J_{\nu} X, Y\right) J_{\nu} Z\right\} \\
& +\sum_{\nu=1}^{3}\left\{g\left(J_{\nu} J Y, Z\right) J_{\nu} J X-g\left(J_{\nu} J X, Z\right) J_{\nu} J Y\right\},
\end{aligned}
$$

where $J_{1}, J_{2}, J_{3}$ is any canonical local basis of $\mathfrak{J}$.

\section{Some fundamental formulas for real hypersurfaces in $G_{2}\left(\mathbb{C}^{m+2}\right)$}

In this section we derive some fundamental formulae which will be used in the proof of our main theorem. Let $M$ be a real hypersurface of $G_{2}\left(\mathbb{C}^{m+2}\right)$, that is, a hypersurface of $G_{2}\left(\mathbb{C}^{m+2}\right)$ with real codimension one. The induced Riemannian metric on $M$ will also be denoted by $g$, and $\nabla$ denotes the Riemannian connection of $(M, g)$. Let $N$ be a local unit normal field of $M$ and $A$ the shape operator of $M$ with respect to $N$.

The Kähler structure $J$ of $G_{2}\left(\mathbb{C}^{m+2}\right)$ induces on $M$ an almost contact metric structure $(\phi, \xi, \eta, g)$. More explicitly, we can define a tensor field $\phi$ of type $(1,1)$, a vector field $\xi$ and its dual 1-form $\eta$ on $M$ by $g(\phi X, Y)=g(J X, Y)$ and $\eta(X)=g(\xi, X)$ for any tangent vector fields $X$ and $Y$ on $M$. Then they 
satisfy the following

$$
\phi^{2} X=-X+\eta(X) \xi, \phi \xi=0, \eta(\phi X)=0 \text { and } \eta(\xi)=1
$$

for any tangent vector field $X$.

Furthermore, let $J_{1}, J_{2}, J_{3}$ be a canonical local basis of $\mathfrak{J}$. Then each $J_{\nu}$ induces an almost contact metric structure $\left(\phi_{\nu}, \xi_{\nu}, \eta_{\nu}, g\right)$ on $M$ in such a way that a tensor field $\phi_{\nu}$ of type $(1,1)$, a vector field $\xi_{\nu}$ and its dual 1-form $\eta_{\nu}$ on $M$ defined by $g\left(\phi_{\nu} X, Y\right)=g\left(J_{\nu} X, Y\right)$ and $\eta_{\nu}(X)=g\left(\xi_{\nu}, X\right)$ for any tangent vector fields $X$ and $Y$ on $M$. Then they also satisfy the following

$$
\phi_{\nu}^{2} X=-X+\eta_{\nu}(X) \xi_{\nu}, \phi_{\nu} \xi_{\nu}=0, \eta_{\nu}\left(\phi_{\nu} X\right)=0 \text { and } \eta_{\nu}\left(\xi_{\nu}\right)=1
$$

for any vector field $X$ tangent to $M$ and $\nu=1,2,3$.

Using the above expression (1.2) for the curvature tensor $\bar{R}$, the equations of Gauss and Codazzi are respectively given by

$$
\begin{aligned}
R(X, Y) Z= & g(Y, Z) X-g(X, Z) Y \\
& +g(\phi Y, Z) \phi X-g(\phi X, Z) \phi Y-2 g(\phi X, Y) \phi Z \\
& +\sum_{\nu=1}^{3}\left\{g\left(\phi_{\nu} Y, Z\right) \phi_{\nu} X-g\left(\phi_{\nu} X, Z\right) \phi_{\nu} Y-2 g\left(\phi_{\nu} X, Y\right) \phi_{\nu} Z\right\} \\
& +\sum_{\nu=1}^{3}\left\{g\left(\phi_{\nu} \phi Y, Z\right) \phi_{\nu} \phi X-g\left(\phi_{\nu} \phi X, Z\right) \phi_{\nu} \phi Y\right\} \\
& -\sum_{\nu=1}^{3}\left\{\eta(Y) \eta_{\nu}(Z) \phi_{\nu} \phi X-\eta(X) \eta_{\nu}(Z) \phi_{\nu} \phi Y\right\} \\
& -\sum_{\nu=1}^{3}\left\{\eta(X) g\left(\phi_{\nu} \phi Y, Z\right)-\eta(Y) g\left(\phi_{\nu} \phi X, Z\right)\right\} \xi_{\nu} \\
& +g(A Y, Z) A X-g(A X, Z) A Y,
\end{aligned}
$$

and

$$
\begin{aligned}
\left(\nabla_{X} A\right) Y-\left(\nabla_{Y} A\right) X= & \eta(X) \phi Y-\eta(Y) \phi X-2 g(\phi X, Y) \xi \\
& +\sum_{\nu=1}^{3}\left\{\eta_{\nu}(X) \phi_{\nu} Y-\eta_{\nu}(Y) \phi_{\nu} X-2 g\left(\phi_{\nu} X, Y\right) \xi_{\nu}\right\} \\
& +\sum_{\nu=1}^{3}\left\{\eta_{\nu}(\phi X) \phi_{\nu} \phi Y-\eta_{\nu}(\phi Y) \phi_{\nu} \phi X\right\} \\
& +\sum_{\nu=1}^{3}\left\{\eta(X) \eta_{\nu}(\phi Y)-\eta(Y) \eta_{\nu}(\phi X)\right\} \xi_{\nu}
\end{aligned}
$$

where $R$ denotes the curvature tensor of a real hypersurface $M$ in $G_{2}\left(\mathbb{C}^{m+2}\right)$. 
The following identities can be proved in a straightforward method and will be used frequently in subsequent calculations (See [8] and [9]):

$$
\begin{aligned}
& \phi_{\nu+1} \xi_{\nu}=-\xi_{\nu+2}, \quad \phi_{\nu} \xi_{\nu+1}=\xi_{\nu+2}, \\
& \phi \xi_{\nu}=\phi_{\nu} \xi, \quad \eta_{\nu}(\phi X)=\eta\left(\phi_{\nu} X\right), \\
& \phi_{\nu} \phi_{\nu+1} X=\phi_{\nu+2} X+\eta_{\nu+1}(X) \xi_{\nu}, \\
& \phi_{\nu+1} \phi_{\nu} X=-\phi_{\nu+2} X+\eta_{\nu}(X) \xi_{\nu+1} .
\end{aligned}
$$

Now let us note that

$$
J X=\phi X+\eta(X) N, \quad J N=-\xi,
$$

and

$$
J_{\nu} X=\phi_{\nu} X+\eta_{\nu}(X) N, \quad J_{\nu} N=-\xi_{\nu}, \quad \nu=1,2,3
$$

for any tangent vector $X$ of a real hypersurface $M$ in $G_{2}\left(\mathbb{C}^{m+2}\right)$, where $N$ denotes a normal vector of $M$ in $G_{2}\left(\mathbb{C}^{m+2}\right)$. Then from these and the formulae (1.1) and (2.3) we have that

$$
\begin{aligned}
\left(\nabla_{X} \phi\right) Y= & \eta(Y) A X-g(A X, Y) \xi, \quad \nabla_{X} \xi=\phi A X, \\
\nabla_{X} \xi_{\nu}= & q_{\nu+2}(X) \xi_{\nu+1}-q_{\nu+1}(X) \xi_{\nu+2}+\phi_{\nu} A X, \\
\left(\nabla_{X} \phi_{\nu}\right) Y= & -q_{\nu+1}(X) \phi_{\nu+2} Y+q_{\nu+2}(X) \phi_{\nu+1} Y+\eta_{\nu}(Y) A X \\
& -g(A X, Y) \xi_{\nu} .
\end{aligned}
$$

Summing up these formulae, we find the following

$$
\begin{aligned}
\nabla_{X}\left(\phi_{\nu} \xi\right)= & \nabla_{X}\left(\phi \xi_{\nu}\right) \\
= & \left(\nabla_{X} \phi\right) \xi_{\nu}+\phi\left(\nabla_{X} \xi_{\nu}\right) \\
= & q_{\nu+2}(X) \phi_{\nu+1} \xi-q_{\nu+1}(X) \phi_{\nu+2} \xi+\phi_{\nu} \phi A X \\
& -g(A X, \xi) \xi_{\nu}+\eta\left(\xi_{\nu}\right) A X .
\end{aligned}
$$

Moreover, from $J J_{\nu}=J_{\nu} J, \nu=1,2,3$, it follows that

$$
\phi \phi_{\nu} X=\phi_{\nu} \phi X+\eta_{\nu}(X) \xi-\eta(X) \xi_{\nu} .
$$

On the other hand, using the fact, $A \xi=\alpha \xi$, Berndt and the second author gave the following lemma (See [4]):

Lemma 2.1. If $M$ is a connected orientable Hopf real hypersurface in $G_{2}\left(\mathbb{C}^{m+2}\right)$, then

$$
\begin{aligned}
& \alpha g((A \phi+\phi A) X, Y)-2 g(A \phi A X, Y)+2 g(\phi X, Y) \\
= & 2 \sum_{\nu=1}^{3}\left\{\eta_{\nu}(X) \eta_{\nu}(\phi Y)-\eta_{\nu}(Y) \eta_{\nu}(\phi X)-g\left(\phi_{\nu} X, Y\right) \eta_{\nu}(\xi)\right. \\
& \left.-2 \eta(X) \eta_{\nu}(\phi Y) \eta_{\nu}(\xi)+2 \eta(Y) \eta_{\nu}(\phi X) \eta_{\nu}(\xi)\right\}
\end{aligned}
$$

for all vector fields $X$ and $Y$ on $M$. 


\section{Proof of Main Theorem}

Let $M$ be a connected orientable Hopf real hypersurface in $G_{2}\left(\mathbb{C}^{m+2}\right)$. Now let us denote by the distribution $\mathfrak{D}$ the orthogonal complement of the distribution $\mathfrak{D}^{\perp}=\operatorname{Span}\left\{\xi_{1}, \xi_{2}, \xi_{3}\right\}$ such that $T_{x} M=\mathfrak{D} \oplus \mathfrak{D}^{\perp}$ for any point $x \in M$.

In order to prove our Main Theorem in the introduction we give a key proposition as follows:

Proposition 3.1. Let $M$ be a connected orientable Hopf real hypersurface in $G_{2}\left(\mathbb{C}^{m+2}\right)$. If the Reeb vector $\xi$ belongs to the distribution $\mathfrak{D}$, then the distribution $\mathfrak{D}$ is invariant under the shape operator $A$ of $M$, that is, $g\left(A \mathfrak{D}, \mathfrak{D}^{\perp}\right)=0$.

Proof. To prove this it suffices to show that $g\left(A \mathfrak{D}, \xi_{\nu}\right)=0, \nu=1,2,3$. In order to do this, we put

$$
\mathfrak{D}=[\xi] \oplus\left[\phi_{1} \xi, \phi_{2} \xi, \phi_{3} \xi\right] \oplus \mathfrak{D}_{0}
$$

where the distribution $\mathfrak{D}_{0}$ is an orthogonal complement of $[\xi] \oplus\left[\phi_{1} \xi, \phi_{2} \xi, \phi_{3} \xi\right]$ in the distribution $\mathfrak{D}$ of the tangent space $T_{x} M, x \in M$, of $M$ in $G_{2}\left(\mathbb{C}^{m+2}\right)$. First, from the assumption $\xi \in \mathfrak{D}$ we know $g\left(A \xi, \xi_{\nu}\right)=0, \nu=1,2,3$, because we have assumed that $M$ is Hopf. Next we assert the formula $g\left(A \phi_{i} \xi, \xi_{\nu}\right)=0$ for $i, \nu=1,2,3$. In fact, by using (2.5) and $\xi \in \mathfrak{D}$ we have the following:

$$
\begin{aligned}
g\left(A \phi_{i} \xi, \xi_{\nu}\right) & =-g\left(\phi A \xi_{\nu}, \xi_{i}\right) \\
& =-g\left(\nabla_{\xi_{\nu}} \xi, \xi_{i}\right) \\
& =g\left(\xi, \nabla_{\xi_{\nu}} \xi_{i}\right) \\
& =g\left(\xi, q_{i+2}\left(\xi_{\nu}\right) \xi_{i+1}-q_{i+1}\left(\xi_{\nu}\right) \xi_{i+2}+\phi_{i} A \xi_{\nu}\right) \\
& =g\left(\xi, \phi_{i} A \xi_{\nu}\right) \\
& =-g\left(A \phi_{i} \xi, \xi_{\nu}\right)
\end{aligned}
$$

which gives our assertion (See [6], page 1127). Finally, we consider for the case $X \in \mathfrak{D}_{0}$. From (2.9) in above Lemma 2.1 , we have

$$
\begin{aligned}
\alpha A \phi X & +\alpha \phi A X-2 A \phi A X+2 \phi X \\
=2 \sum_{\nu=1}^{3}\{- & \eta_{\nu}(X) \phi_{\nu} \xi-\eta_{\nu}(\phi X) \xi_{\nu}-\eta_{\nu}(\xi) \phi_{\nu} X \\
& \left.+2 \eta(X) \eta_{\nu}(\xi) \phi_{\nu} \xi+2 \eta_{\nu}(\phi X) \eta_{\nu}(\xi) \xi\right\}
\end{aligned}
$$

for any tangent vector field $X \in T_{x} M, x \in M$. From now on, we show that $g\left(A X, \xi_{\nu}\right)=0$ for any $X \in \mathfrak{D}_{0}$. In order to do this, we restrict $X \in T_{x} M$, $x \in M$ to $X \in \mathfrak{D}_{0}$ unless otherwise stated. Now by taking $\phi$ into above equation and using the fact $A \xi=\alpha \xi$ we get

$$
\alpha \phi A \phi X-\alpha A X-2 \phi A \phi A X-2 X=0
$$

for any $X \in \mathfrak{D}_{0}$.

Taking inner product in (3.1) with $\xi_{\mu}$ we have

$$
\alpha g\left(\phi A \phi X, \xi_{\mu}\right)-\alpha g\left(A X, \xi_{\mu}\right)-2 g\left(\phi A \phi A X, \xi_{\mu}\right)=0,
$$


that is,

$$
\alpha g\left(A X, \xi_{\mu}\right)=\alpha g\left(\phi A \phi X, \xi_{\mu}\right)-2 g\left(\phi A \phi A X, \xi_{\mu}\right) \quad \text { for } X \in \mathfrak{D}_{0} .
$$

On the other hand, since $g\left(\phi A \phi X, \xi_{\mu}\right)=g\left(\nabla_{\phi X} \xi, \xi_{\mu}\right)=-g\left(\xi, \nabla_{\phi X} \xi_{\mu}\right)$, we have

$$
g\left(\phi A \phi X, \xi_{\mu}\right)=-g\left(\xi, \phi_{\mu} A \phi X\right)=-g\left(\xi_{\mu}, \phi A \phi X\right)
$$

by virtue of (2.3) and (2.5). Accordingly, we get

$$
g\left(\phi A \phi X, \xi_{\mu}\right)=0
$$

for any $X \in \mathfrak{D}_{0}$.

Next let us show that $g\left(\phi A \phi A X, \xi_{\mu}\right)=0$ for any $X \in \mathfrak{D}_{0}$.

In fact, (2.4) and (2.5) give

$$
\begin{aligned}
g\left(\phi A \phi A X, \xi_{\mu}\right) & =g\left(\nabla_{\phi A X} \xi, \xi_{\mu}\right)=-g\left(\xi, \nabla_{\phi A X} \xi_{\mu}\right) \\
& =-g\left(\xi, \phi_{\mu} A \phi A X\right)=-g\left(\xi_{\mu}, \phi A \phi A X\right),
\end{aligned}
$$

which gives our assertion. Thus, from (3.2) we know that

$$
\alpha g\left(A X, \xi_{\mu}\right)=0 \text { for any } X \in \mathfrak{D}_{0} .
$$

Then we are able to divide two cases as follows:

Case 1. $\quad \alpha \neq 0$

From (3.3) the conclusion is obvious.

Case 2. $\quad \alpha=0$

From an assumption, $\alpha=0$, together with (3.1), we have

$$
X=-\phi A \phi A X \quad \text { for any } X \in \mathfrak{D}_{0} .
$$

From this, let us apply the shape operator $A$. Then it follows that

$$
A X=-A \phi A \phi A X \quad \text { for any } X \in \mathfrak{D}_{0} .
$$

Taking an inner product of (3.4) and $\xi_{\mu}$, we have

$$
g\left(A X, \xi_{\mu}\right)=-g\left(A \phi A \phi A X, \xi_{\mu}\right) \text { for any } X \in \mathfrak{D}_{0} .
$$

On the other hand, we know the following

$$
g\left(A \phi A \phi A X, \xi_{\mu}\right)=-g\left(A \phi A X, \phi A \xi_{\mu}\right)=-g\left(A \phi A X, \nabla_{\xi_{\mu}} \xi\right) .
$$

Then it follows that

$$
\begin{aligned}
g\left(A \phi A \phi A X, \xi_{\mu}\right)=- & g\left(A \phi A X, \nabla_{\xi_{\mu}} \xi\right) \\
= & g\left(\left(\nabla_{\xi_{\mu}} A\right) \phi A X, \xi\right)+g\left(A\left(\nabla_{\xi_{\mu}} \phi\right) A X, \xi\right) \\
& +g\left(A \phi\left(\nabla_{\xi_{\mu}} A\right) X, \xi\right)+g\left(A \phi A\left(\nabla_{\xi_{\mu}} X\right), \xi\right),
\end{aligned}
$$

where we have used $g(A \phi A X, \xi)=0$. From this, together with $A \xi=0$, it follows that

$$
g\left(A \phi A \phi A X, \xi_{\mu}\right)=g\left(\left(\nabla_{\xi_{\mu}} A\right) \phi A X, \xi\right) .
$$

On the other hand, by using the equation of Codazzi in Section 2, we have the following: 
Lemma 3.2. $g\left(\left(\nabla_{\xi_{\mu}} A\right) \phi A X, \xi\right)=-g\left(A \xi_{\mu}, \phi A \phi A X\right)-4 g\left(A X, \xi_{\mu}\right)$.

Proof. By the Codazzi equation we know

$$
\begin{aligned}
\left(\nabla_{\xi_{\mu}} A\right) \phi A X= & \left(\nabla_{\phi A X} A\right) \xi_{\mu}+\eta\left(\xi_{\mu}\right) \phi^{2} A X-\eta(\phi A X) \phi \xi_{\mu}-2 g\left(\phi \xi_{\mu}, \phi A X\right) \xi \\
& +\sum_{\nu=1}^{3}\left\{\eta_{\nu}\left(\xi_{\mu}\right) \phi_{\nu} \phi A X-\eta_{\nu}(\phi A X) \phi_{\nu} \xi_{\mu}-2 g\left(\phi_{\nu} \xi_{\mu}, \phi A X\right) \xi_{\nu}\right\} \\
& +\sum_{\nu=1}^{3}\left\{\eta_{\nu}\left(\phi \xi_{\mu}\right) \phi_{\nu} \phi^{2} A X-\eta_{\nu}\left(\phi^{2} A X\right) \phi_{\nu} \phi \xi_{\mu}\right\} \\
& +\sum_{\nu=1}^{3}\left\{\eta\left(\xi_{\mu}\right) \eta_{\nu}\left(\phi^{2} A X\right)-\eta(\phi A X) \eta_{\nu}\left(\phi \xi_{\mu}\right)\right\} \xi_{\nu} \\
= & \left(\nabla_{\phi A X} A\right) \xi_{\mu}-2 g\left(\xi_{\mu}, A X\right) \xi+\phi_{\mu} \phi A X+\sum_{\nu=1}^{3} g\left(\phi \xi_{\nu}, A X\right) \phi_{\nu} \xi_{\mu} \\
& +2 \sum_{\nu=1}^{3} g\left(\phi \phi_{\nu} \xi_{\mu}, A X\right) \xi_{\nu}+\sum_{\nu=1}^{3} \eta_{\nu}(A X) \phi_{\nu} \phi \xi_{\mu} .
\end{aligned}
$$

From this, taking an inner product with $\xi$ and using the fact that $\phi \phi_{\mu} \xi=-\xi_{\mu}$, we have

$$
\begin{aligned}
g\left(\left(\nabla_{\xi_{\mu}} A\right) \phi A X, \xi\right)= & g\left(\left(\nabla_{\phi A X} A\right) \xi_{\mu}, \xi\right)-2 g\left(\xi_{\mu}, A X\right)+g\left(\phi_{\mu} \phi A X, \xi\right) \\
& +\sum_{\nu=1}^{3} g\left(\phi \xi_{\nu}, A X\right) g\left(\phi_{\nu} \xi_{\mu}, \xi\right)+2 \sum_{\nu=1}^{3} g\left(\phi \phi_{\nu} \xi_{\mu}, A X\right) g\left(\xi_{\nu}, \xi\right) \\
& +\sum_{\nu=1}^{3} \eta_{\nu}(A X) g\left(\phi_{\nu} \phi \xi_{\mu}, \xi\right) \\
= & g\left(\left(\nabla_{\phi A X} A\right) \xi_{\mu}, \xi\right)-4 g\left(A X, \xi_{\mu}\right) .
\end{aligned}
$$

On the other hand, since $g\left(A \xi_{\mu}, \xi\right)=g\left(\xi_{\mu}, A \xi\right)=\alpha g\left(\xi_{\mu}, \xi\right)$ and $\alpha=0$, we have

$$
\begin{aligned}
g\left(\left(\nabla_{\phi A X} A\right) \xi_{\mu}, \xi\right) & =-g\left(A\left(\nabla_{\phi A X} \xi_{\mu}\right), \xi\right)-g\left(A \xi_{\mu}, \phi A \phi A X\right) \\
& =-\alpha g\left(\nabla_{\phi A X} \xi_{\mu}, \xi\right)-g\left(A \xi_{\mu}, \phi A \phi A X\right) \\
& =-g\left(A \xi_{\mu}, \phi A \phi A X\right) .
\end{aligned}
$$

Therefore we have

$$
g\left(\left(\nabla_{\xi_{\mu}} A\right) \phi A X, \xi\right)=-g\left(A \xi_{\mu}, \phi A \phi A X\right)-4 g\left(A X, \xi_{\mu}\right)
$$

for any $X \in \mathfrak{D}_{0}$.

Consequently, from (3.6), Lemma 3.2, the symmetry of the shape operator $A$, and together with the fact that $A \xi=0$, we get

$$
g\left(A \phi A \phi A X, \xi_{\mu}\right)=-2 g\left(A X, \xi_{\mu}\right) .
$$


From (3.5) and (3.7) for $\alpha=0$, we have $g\left(A X, \xi_{\mu}\right)=0$ for any tangent vector field $X$ belongs to the distribution $\mathfrak{D}_{0}$.

Then summing up all situation mentioned above we conclude that the distribution $\mathfrak{D}$ is invariant under the shape operator of $M$ if the Reeb vector $\xi$ belong to the distribution $\mathfrak{D}$.

Then by Proposition 3.1 and Theorem $A$ we know that a Hopf real hypersurface $M$ in $G_{2}\left(\mathbb{C}^{m+2}\right)$ with the Reeb vector $\xi$ belongs to the distribution $\mathfrak{D}$ is congruent to a tube over a totally geodesic $G_{2}\left(\mathbb{C}^{m+1}\right)$ in $G_{2}\left(\mathbb{C}^{m+2}\right)$ or a tube over a totally geodesic $\mathbb{Q} P^{n}, m=2 n$, in $G_{2}\left(\mathbb{C}^{m+1}\right)$. But in [3] it was known that the Reeb vector $\xi$ of type $A$ in the first case belongs to the distribution $\mathfrak{D}^{\perp}$. From this we complete the proof of our main theorem in the introduction.

Acknowledgments. The present authors would like to express their deep gratitude to the referee for his/her careful reading of our manuscript and valuable comments to develop the first version.

\section{References}

[1] J. Berndt, Riemannian geometry of complex two-plane Grassmannians, Rend. Sem. Mat. Univ. Politec. Torino 55 (1997), no. 1, 19-83.

[2] _ Real hypersurfaces with constant principal curvatures in complex space forms, Proceedings of the Tenth International Workshop on Differential Geometry, 1-12, Kyungpook Nat. Univ., Taegu, 2006.

[3] J. Berndt and Y. J. Suh, Real hypersurfaces in complex two-plane Grassmannians, Monatsh. Math. 127 (1999), no. 1, 1-14.

[4] _ Real hypersurfaces with isometric Reeb flow in complex two-plane Grassmannians, Monatsh. Math. 137 (2002), no. 2, 87-98.

[5] T. E. Cecil and P. J. Ryan, Focal sets and real hypersurfaces in complex projective space, Trans. Amer. Math. Soc. 269 (1982), no. 2, 481-499.

[6] I. Jeong and Y. J. Suh, Real hypersurfaces in complex two-plane Grassmannians with Lie $\xi$-parallel normal Jacobi operator, J. Korean Math. Soc. 45 (2008), no. 4, 1113-1133.

[7] M. Kimura, Real hypersurfaces and complex submanifolds in complex projective space, Trans. Amer. Math. Soc. 296 (1986), no. 1, 137-149.

[8] Y. J. Suh, Real hypersurfaces in complex two-plane Grassmannians with commuting shape operator, Bull. Austral. Math. Soc. 68 (2003), no. 3, 379-393.

[9] , Real hypersurfaces of type B in complex two-plane Grassmannians, Monatsh. Math. 147 (2006), no. 4, 337-355.

[10] R. Takagi, On homogeneous real hypersurfaces in a complex projective space, Osaka J. Math. 10 (1973), 495-506.

[11] K. Yano and M. Kon, CR Submanifolds of Kaehlerian and Sasakian Manifolds, Progress in Mathematics, 30. Birkhauser, Boston, Mass., 1983.

HyUnJin Lee

Graduate School of Electrical Engineering \& Computer Science

KYUNGPOOK NATIONAL University

TAEGU 702-701, KOREA

E-mail address: lhjibis@hanmail.net 
YOUNG JiN SuH

Department of Mathematics

KyungPoOK National University

TAEgu 702-701, Korea

E-mail address: yjsuh@knu.ac.kr 